\title{
Li Norm Solution of Overdetermined System of Linear Equations
}

\author{
Bijan Bidabad \\ B.A., M.Sc., Ph.D., Post-Doc. \\ Professor \\ Economics and Chief Islamic Banking Advisor \\ Bank Melli, Iran \\ E-mail:bijan@bidabad.com
}

\begin{abstract}
In this paper, three algorithms for weighted median, simple linear, and multiple $m$ parameters $\mathrm{L}_{\mathrm{I}}$ norm regressions are introduced. The corresponding computer programs are also included.
\end{abstract}

\section{Keywords: Li norm, Regression, Algorithm, Computer program}

\section{Introduction}

Li norm criterion is going to find its place in scientific analysis. Since it is not computationally comparable with other criteria such as $\mathrm{L}_{2}$ norm, it needs more work to make it a hand tool. The closed form of the solution of the $\mathrm{L}_{\mathrm{i}}$ norm estimator has not been derived yet, and therefore, makes further inferences of the properties of this estimator difficult. Any attempt to give efficient computational algorithms which may introduce significant insight into the different characteristics of the problem is desirable. In this regard, Bidabad (1989a,b) gives a general procedure to solve the $\mathrm{L}_{\mathrm{I}}$ norm linear regression problem. The proposed algorithms are based on a special descent method and use a discrete differentiation technique. Primary designs of the algorithms have been discussed by Bidabad (1987a,b,88a,b). By manipulating the algorithms, more efficient ones were introduced by Bidabad (I989a,b), which has been shown to have better performance than other existing algorithms.

Consider the following regression model,

$$
y_{i}=\sum_{j=I}^{m} \beta_{j} x_{i j}+u_{i} \quad i=I, \ldots, n
$$

where $\beta_{j}, j=I, \ldots, m$ are unknown population parameters to be estimated, $y_{i}, x_{i j}$, and $u_{i}$ are dependent, independent and random error variables respectively. We wish to estimate $\beta_{j}$ 's by minimizing the sum of absolute errors given by the following expression:

$$
\mathrm{S}=\sum_{\mathrm{i}=\mathrm{I}}^{\mathrm{n}}\left|\mathrm{u}_{\mathrm{i}}^{\wedge}\right|=\sum_{\mathrm{i}=\mathrm{I}}^{\mathrm{n}}\left|\mathrm{y}_{\mathrm{i}}-\sum_{\mathrm{j}=\mathrm{I}}^{\mathrm{m}} \beta_{\mathrm{j}}^{\wedge} \mathrm{x}_{\mathrm{ji}}\right|
$$

where $\beta_{j} \wedge$ is the estimated value of $\beta_{j}$. When $\mathrm{m}=\mathrm{I}$, we are confronted with a weighted median problem.

2. Weighted median computation (restricted one parameter model)

Let us now consider a simple restricted linear model in which $\mathrm{m}=\mathrm{I}$ namely,

$y_{i}=\beta_{I X i I}+u_{i}$

For the model given by (3), the LI norm objective function $\mathrm{S}$ to be minimized will be,

$$
\mathrm{S}=\Sigma\left|\mathrm{y}_{\mathrm{i}}-\beta_{\mathrm{IX} \text { XI }}\right|=\Sigma\left|\mathrm{x}_{\mathrm{iI}}\right|\left|\mathrm{y}_{\mathrm{i}} / \mathrm{x}_{\mathrm{iI}}-\beta\right|
$$




$$
\mathrm{i}=\mathrm{I} \quad \mathrm{i}=\mathrm{I}
$$

Two series of computations are necessary to compute the weighted median. One sorting algorithm is essential to sort the ratio array $\left(y_{i} / x_{i I}\right)$ and restoring the corresponding subscripts for the second part of the calculation to find the left and right weights $\left(\left|\mathrm{x}_{\mathrm{iI}}\right|\right)$ sequences.

Efficient sorting algorithms exist for the first part of the computation. The algorithms 'quicksort' of Hoare (1961,62), 'quickersort' of Scowen (1965) and 'sort' of Singleton (1969) have desirable performances and efficiencies. For the second part of the computation, there is no special purpose procedure, but Bloomfield and Steiger (1980) used the partial sorting of Chambers (I97I) to give an efficient way to combine the two steps of sorting and finding the optimal observation. The superiority of this procedure is in sorting the smaller segments of the array rather than all its elements. With some modification, this procedure is used by Bidabad (1989a,b). The procedure can be stated as the following function.

\section{FUNCTION LWMED (n,ys,w,1)}

Step 0) Initialization.

Real: ys(n), w(n).

Integer: $1(\mathrm{n})$, hi.

Set: ii $=0$, shi $=0$, slo $=0, \mathrm{sz}=0, \mathrm{sp}=0, \mathrm{sn}=0$.

Step I) Compute left, the middle and right sum of weights.

Do loop for $i=I, n: w(i)=|w(i)|$; if $y s(i)<0$, then $s n=s n+w(i)$, if $y s(i)>0$, then $s p=s p+w(i)$, if $y s(i)=0$ then $s z=s z+w(i)$; end do.

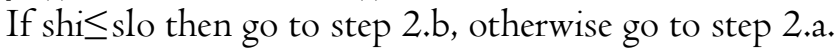

Step 2) Assign subscripts for arrays.

a. Let: shi $=0$.

Do loop for $\mathrm{i}=\mathrm{I}$, n: if $y s(\mathrm{i}) \leq 0$ go to continue, otherwise $i \mathrm{ii}=\mathrm{ii}+\mathrm{I}, \mathrm{l}(\mathrm{ii})=\mathrm{i}$, continue, end do.

Go to step 2.c.

b. Let: $\mathrm{slo}=0$.

Do loop for $i=I$, n: if $y s(i)>0$ go to continue, otherwise $i i=i i+I, 1(i i)=i$, continue, end do.

c. Let: $1 \mathrm{o}=\mathrm{I}$, hi $=$ ii.

Step 3) Check for solution.

If $\mathrm{hi}>\mathrm{lo}+\mathrm{I}$ then go to step 4 , otherwise lwmed $=1(1 \mathrm{o})$.

If $l_{\mathrm{o}}=$ hi return, otherwise if $y s(1(\mathrm{lo})) \leq y s(1(\mathrm{hi}))$ go to step 3 .a, otherwise $1 \mathrm{t}=1(\mathrm{lo}), 1(\mathrm{lo})=1(\mathrm{hi})$,

1 (hi) $=1 \mathrm{t}$, lwmed $=1(\mathrm{lo})$.

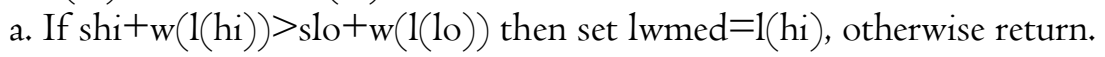

Step 4) Divide the string into two halves then sort.

Set: $\mathrm{mid}=(1 \mathrm{o}+\mathrm{hi}) / 2$, lop $=1 \mathrm{o}+\mathrm{I}, \mathrm{lt}=1(\mathrm{mid}), 1(\mathrm{mid})=1(\mathrm{lop}), 1(\mathrm{lop})=1 \mathrm{t}$.

a. If $y s(1($ lop $)) \leq y s(1($ hi $))$ then go to step $4 . b$, otherwise $1 t=1($ lop $), 1($ lop $)=1$ (hi), 1 (hi) $=1$ t.

b. If $y s(1(10)) \leq y s(1(h i))$ then go to step 4 .c, otherwise $1 t=1(10), 1(l o)=1(h i), 1(h i)=l t$.

c. If $y s(1($ lop $)) \leq y s(1($ lo $))$ then go to step 5 , otherwise $1 t=1($ lop $), 1($ lop $)=1(1 \mathrm{o}), 1(1 \mathrm{o})=1$.

Step 5) Compute the accumulation of weights.

Let: lwmed $=1(10), i=l o p, j=h i, x t=y s(1 w m e d)$, tlo=slo, thi $=$ shi.

a. Set: $\mathrm{tlo}_{\mathrm{o}}=\mathrm{tlo}_{\mathrm{w}}(\mathrm{l}(\mathrm{i})), \mathrm{i}=\mathrm{i}+\mathrm{I}$.

If $y s(1(i))<x t$ then go to step 5.a, otherwise go to step 5.b.

b. Let: thi $=\operatorname{thi}_{w}(1(j)), j=j-I$.

If $y s(1(j))>x t$ then go to step $5 . b$, otherwise if $j \leq i$ then go to step 6 , otherwise $1 t=1(i), 1(i)=1(j)$,

$1(j)=1$, go to step 5.a.

Step 6) Test for solution. 
Let: test $={ }_{w}\left(1_{\text {wmed }}\right)$.

If $i \neq j$ then go to step 6 .a, otherwise test $=$ test $+w(1(i)), i=i+I, j=j-I$.

a. If test $\geq \mid$ thi-tlo $\mid$ then return, otherwise, if tlo $>$ thi then step $6 . \mathrm{b}$, otherwise $\mathrm{slo}=\mathrm{tlo}+$ test, $1 \mathrm{o}=\mathrm{i}$, go to step 3 .

b. Let: shi $=$ thi + test, $l_{\mathrm{o}}=$ lop, $\mathrm{hi}=\mathrm{j}$.

Go to step 3.

END

\section{Unrestricted simple linear regression}

Let us now consider a simple unrestricted linear model in which $\mathrm{m}=2$ and $\mathrm{x}_{\mathrm{Ii}}=\mathrm{I}$ for all $\mathrm{i}=\mathrm{I}, \ldots, \mathrm{n}$; namely,

$$
y_{i}=\beta_{1}+\beta_{2 x_{i 2}}+u_{i}
$$

For the model given in (5), the $\mathrm{L}_{1}$ norm objective function $\mathrm{S}$ to be minimized will be,

$$
\mathrm{S}=\sum_{\mathrm{i}=\mathrm{I}}^{\mathrm{n}} \mathrm{y}_{\mathrm{i}}-\beta_{\mathrm{I}}-\beta_{2 \times 2 \mathrm{2}} \mid
$$

\section{PROGRAM BLIS}

Step 0) Initialization.

Parameter: $n$.

Real: $y(n), x 2(n), z(n), w(n)$.

Integer: $1(\mathrm{n})$.

Set: $\mathrm{kI}=$ arbitrary, $\mathrm{kIr}=0, \mathrm{kIs}=0$, iter $=0$.

$\operatorname{Read}(y(i), x 2(i), i=I, n)$

Step I) Compute weights and ratios.

Do loop for $i=I, k I-I: w(i)=x 2(i)-x 2(k I), z(i)=(y(i)-y(k I)) / w(i)$, end do.

Set: $w(k I)=0, z(k I)=0$.

Do loop for $i=k I+I, n: w(i)=x 2(i)-x 2(k I), z(i)=(y(i)-y(k I)) / w(i)$ end do.

Set: iter $=$ iter+I.

Step 2) Compute weighted median.

Let: $1 \mathrm{~m}=\operatorname{LWMED}(\mathrm{n}, \mathrm{z}, \mathrm{w}, 1)$.

Step 3) Check for optimality.

Set: $\mathrm{kIs}=\mathrm{kIr}, \mathrm{kIr}=\mathrm{kI}$.

If $1 \mathrm{~m}=\mathrm{kI}$ s then go to step 4 , otherwise $\mathrm{kI}=\mathrm{lm}$.

Go to step I.

Step 4) Compute the solution.

Let $\mathrm{b} 2=\mathrm{z}(\mathrm{lm}), \mathrm{bI}=\mathrm{y}(\mathrm{kI})-\mathrm{b} 2^{*} \mathrm{x} 2(\mathrm{kI})$.

Print bI, b2, kI, lm, iter.

stop.

END

4. General linear model

For the general $\mathrm{m}$ parameter model, the following algorithm is proposed.

PROGRAM BLI

Step 0) Initialization.

Parameter: $\mathrm{n}, \mathrm{m}, \mathrm{mI}=\mathrm{m}-\mathrm{I}, \mathrm{m} 2=\mathrm{m}-2$. 
Real: $y(n), x(n, m I), x s k(m I), y w(n), x k w(n), w(n), y s(n), x s(n, m I), b(m), x w(n, 2: m I), y s o l(m I)$, $\operatorname{xsol}(\mathrm{mI}, \mathrm{mI})$.

Integer: $1(\mathrm{n}), \mathrm{kk}(\mathrm{mI})$.

Common: /cI/iI,i2.

Read: $(y(i),(x(i, j), j=I, m I), i=I, n)$.

Let: iter $=0, \mathrm{kr}=0, \mathrm{~mm}=\mathrm{I},(\mathrm{kk}(\mathrm{j})=$ arbitrary, $\mathrm{j}=\mathrm{I}, \mathrm{mI})$.

Step I) Refill working arrays.

Do loop for $i=I, n: y s(i)=y(i)$, do loop for $j=I, m I: x s(i, j)=x(i, j)$, end do, end do.

Step 2) Store weights and ratios for next iteration.

Do loop for $i=I, n: w(i)=x k w(i), y s(i)=y w(i)$, do loop for $j=I, m I: x s(i, j)=x w(i, j)$, end do, end do.

Step 3 ) Compute the arguments for weighted median.

a. Set: $\mathrm{jj}=\mathrm{mm}, \mathrm{k}=\mathrm{kk}(\mathrm{ji}), \mathrm{ysk}=\mathrm{ys}(\mathrm{k}), \mathrm{iI}=\mathrm{I}, \mathrm{i} 2=\mathrm{k}-\mathrm{I}$.

Do loop for $j=j j, m I: x s k(j)=x s(k, j)$, end do.

b. Do loop for $\mathrm{j}=\mathrm{ji}, \mathrm{mI}$ : call $\operatorname{COLI}(\operatorname{xsk}(\mathrm{j}), \mathrm{xs}(\mathrm{I}, \mathrm{j}))$ end do.

Call COL2(ysk,ji,w,ys,xs $(\mathrm{I}, \mathrm{ji}))$.

If $i 2=$ no to step $3 . c$; otherwise set: $i \mathrm{I}=\mathrm{k}+\mathrm{I}, \mathrm{i} 2=\mathrm{n}$, go to step $3 . \mathrm{b}$.

c. Set: $w(k)=0$.

If $\mathrm{jj}=\mathrm{mI}$ go to step 4 ; otherwise $\mathrm{i}=\mathrm{I}, \mathrm{i} 2=\mathrm{k}-\mathrm{I}$, go to step $3 . \mathrm{d}$.

d. Do loop for $\mathrm{j}=\mathrm{jj}+\mathrm{I}, \mathrm{mI}$ : call $\operatorname{COL} 3(\mathrm{xs}(\mathrm{I}, \mathrm{j}), \mathrm{xs}(\mathrm{I}, \mathrm{ji}))$, end do.

If $\mathrm{i}=\mathrm{n}$ go to step 3.e; otherwise $\mathrm{iI}=\mathrm{k}+\mathrm{I}, \mathrm{i} 2=\mathrm{n}$, go to step 3.d.

e. If $\mathrm{j} \neq \mathrm{mm} \mathrm{jj}=\mathrm{jj}+\mathrm{I}$, go to step 3 , otherwise do loop for $\mathrm{i}=\mathrm{I}, \mathrm{n}: \mathrm{xkw}(\mathrm{i})={ }_{w}(\mathrm{i}), y w(\mathrm{i})=\mathrm{ys}(\mathrm{i})$; do loop for $\mathrm{j}=\mathrm{jj}+\mathrm{I}, \mathrm{mI}: \mathrm{xw}(\mathrm{i}, \mathrm{j})=\mathrm{xs}(\mathrm{i}, \mathrm{j})$, end do; end do.

Set: $\mathrm{jj}=\mathrm{jj}+\mathrm{I}$, go to step 3 .

Step 4) Compute the weighted median.

Set: $y s(k)=0$, iter $=i t e r+I, 1 m=\operatorname{LWMED}(n, y s, w, 1)$.

Step 5) Test for optimality.

If $1 \mathrm{~m}=\mathrm{kr}$ go to step 5.b; otherwise iopt $=0$ go to step 5.a.

a. If $\mathrm{mm}=\mathrm{mI}$ set $\mathrm{mm}=\mathrm{I}, \mathrm{kr}=\mathrm{kk}(\mathrm{mm}), \mathrm{kk}(\mathrm{mm})=\mathrm{lm}$, go to step $\mathrm{I}$; otherwise set $\mathrm{mm}=\mathrm{mm}+\mathrm{I}$, $\mathrm{kr}=\mathrm{kk}(\mathrm{mm}), \mathrm{kk}(\mathrm{mm})=\mathrm{lm}$, go to step 2 .

b. Set: iopt $=$ iopt + I.

If iopt $\neq \mathrm{mI}$ go to step 5.a, otherwise go to step 6 .

Step 6) Compute the solution.

Set: $b(\mathrm{~m})=y s(\mathrm{~lm})$.

Do loop for $i=I, m I$ : $y \operatorname{sol}(i)=y(k k(i))$; do loop for $j=I, m I: x s o l(i, j)=x(k k(i), j)$, end do; end do.

Set: $\ddot{j j}=\mathrm{I}$.

a. Set: $y$ sk $=y \operatorname{sol}($ ji $)$.

Do loop for $\mathrm{j}=\mathrm{jj}, \mathrm{mI}: \mathrm{xsx}(\mathrm{j})=\mathrm{xsol}(\mathrm{ji}, \mathrm{j})$, end do.

Do loop for $\mathrm{i}=\mathrm{jj}, \mathrm{mI}$ : if $\mathrm{i}=\mathrm{jj}$ go to continue; otherwise ysol( $\mathrm{i})=y \operatorname{sol}(\mathrm{i})$-ysk, do loop for $\mathrm{j}=\mathrm{jj}, \mathrm{mI}$ :

$x \operatorname{xol}(i, j)=x \operatorname{xol}(i, j)-x \operatorname{se}(j)$, end do; $\operatorname{set} y \operatorname{sol}(i)=y \operatorname{sol}(i) / x \operatorname{sol}(i, j i)$, continue, end do.

b. Do loop for $\mathrm{i}=\mathrm{ji}, \mathrm{mI}$ : if $\mathrm{i}=\mathrm{jj}$ go to continue, otherwise, do loop for $\mathrm{j}=\mathrm{jj}+\mathrm{I}, \mathrm{mI}$ :

$\operatorname{xsol}(i, j)=x \operatorname{xol}(i, j) / x s o l(i, j)$, end do; continue; end do.

c. If $\mathrm{jj}=\mathrm{m} 2$ go to step $6 . \mathrm{d}$; otherwise go to step 6.a.

d. Do loop for $\mathrm{i}=\mathrm{I}, \mathrm{m} 2 \mathrm{k} \mathrm{k}=\mathrm{m}-\mathrm{i}, \mathrm{s}=y \operatorname{sol}(\mathrm{k})$; do loop for $\mathrm{j}=\mathrm{k}, \mathrm{mI}, \mathrm{s}=\mathrm{s}-\mathrm{b}(\mathrm{j}+\mathrm{I})^{*} \mathrm{xsol}(\mathrm{k}, \mathrm{j})$ end do, $\mathrm{b}(\mathrm{k})=\mathrm{s}$, end do. Set: $s=y(k k(I))$.

Do loop for $j=I, m I: s=s-b(j+I){ }^{*} x(k k(I), j), b(I)=s$, end do.

Print: $((b(j), j=I, m),(k k(j), j=I, m I), \operatorname{lm}$, iter $)$. 
Stop.

END

The major portion of computation in this program is the transformation of two-dimensional arrays. Passing columns of these arrays to other subroutines which involve only one-dimensional arrays saves the time of computation (see, Barrodale and Roberts (1974)). Subroutine COLI, COL2, and COL3 have been coded to do this task for subtraction, multiplication, and division, and for only division respectively. Function LWMED, which is used to compute the weighted median has been introduced in section 2.I.

\section{SUBROUTINE COLI (vI,v2)}

Step 0) Initialization

Real: v2(I).

Common /cI/iI,i2.

Step I) Subtraction.

Do loop for $i=i I, i 2$ : v2(i) $={ }_{v} 2(i)-v I$, end do.

Return.

END

SUBROUTINE COL2(ysk,ji,vI,ys,v2)

Step 0) Initialization.

Real: vI(I),v2(I),ys(I).

Common /cI/iI,i2.

Step I) Compute weights and ratios.

If $\mathrm{jj} \neq \mathrm{I}$ go to step I.a,; otherwise do loop for $\mathrm{i}=\mathrm{iI}, \mathrm{i} 2 \mathrm{v} \mathrm{vI}(\mathrm{i})=\mathrm{v} 2(\mathrm{i})$,

$y s(i)=(y s(i)-y s k) / v 2(i)$.

Return.

a. Do loop for $i=i I, i 2: v I(i)={ }_{v} I(i)^{*} v 2(i), y s(i)=(y s(i)-y s k) / v 2(i)$, end do.

Return.

END

\section{SUBROUTINE COL3(vI,v2)}

Step 0) Initialization.

Real: vI(I),v2(I),ys(I).

Common /cI/iI,i2.

Step I) Division.

Do loop for $i=i I, i 2: v I(i)={ }_{v} I(i) / v 2(i)$, end do.

Return.

END

5. Computer programs

FUNCTION LWMED(N,YS,W,L)

C N Number of observations (input).

C YS(I) The array to be sorted (yi/xiI) (input).

C $\mathrm{W}(\mathrm{N})$ The weight array (xiI) (input).

C L The index of location of weighted median in the unsorted arrays (outpot). REAL YS(N),W(N)

INTEGER L(N),HI 


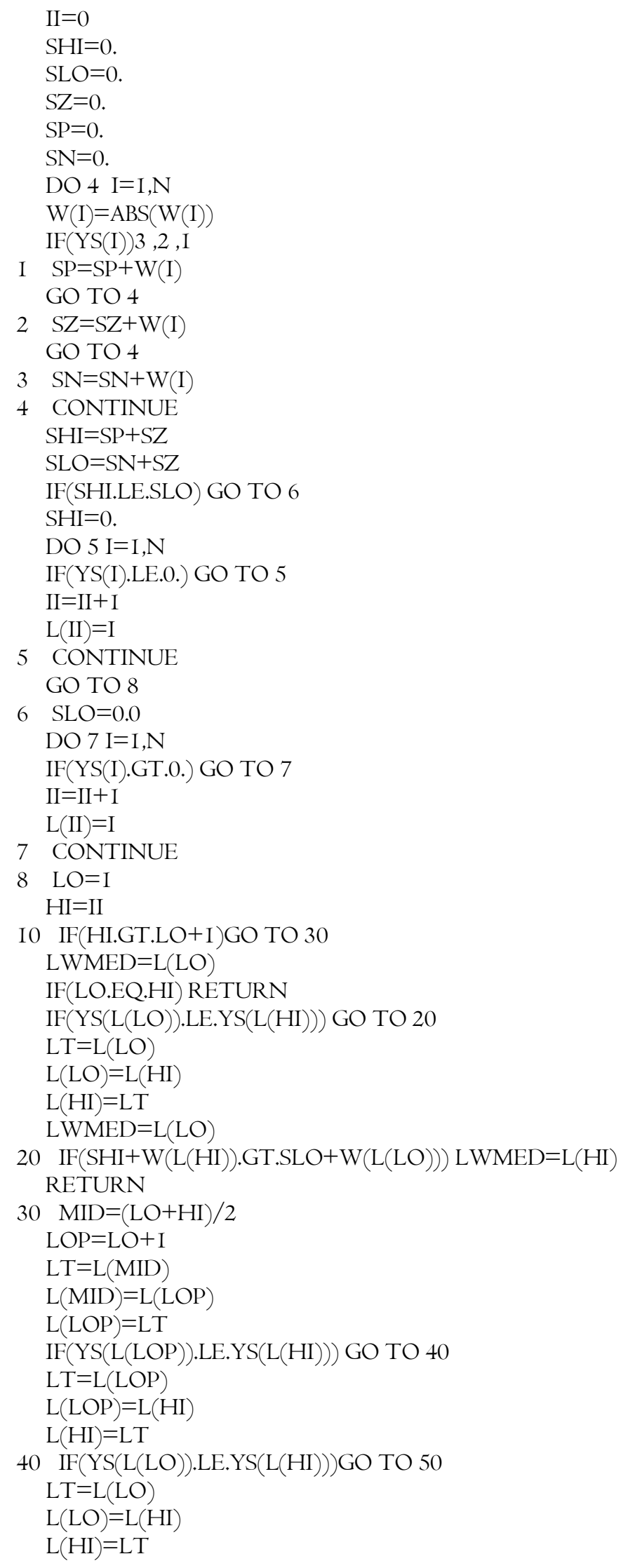




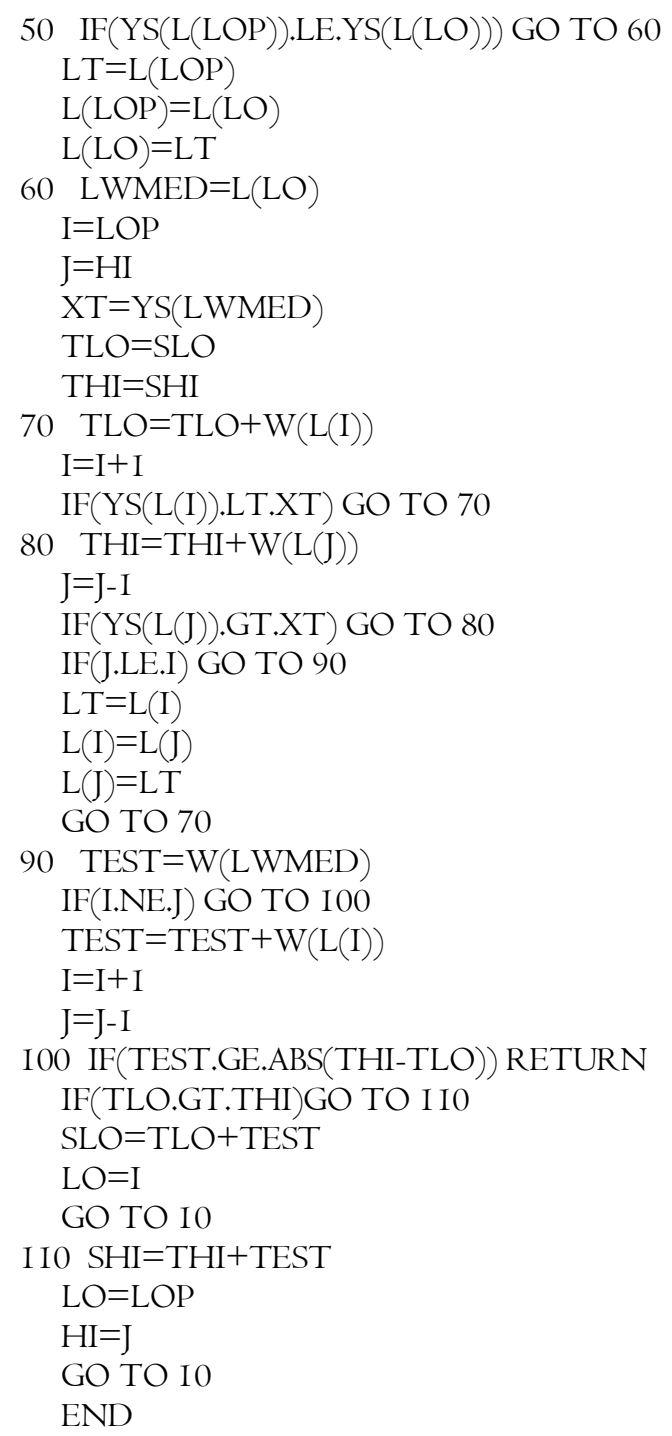

\section{PROGRAM BLIS}

C N Number of observation (input).

C $\mathrm{Y}(\mathrm{N})$ Dependent variable observations array (yi) (input).

C X2(N) Independent variable observations array (x2i) (input).

C $\mathrm{Z}(\mathrm{N})$ Working array for (yi/xi2).

C W(N) Working array for index of sorted array of (yi/xiI).

C L(N) Working array for weights (xiI). PARAMETER $(\mathrm{N}=\mathrm{IOO0})$ DIMENSION Y(N),X2(N),Z(N),W(N),L(N) DO IO I $=I, N$

I0 $\operatorname{READ}(5,20) \mathrm{Y}(\mathrm{I}), \mathrm{X} 2(\mathrm{I})$

20 FORMAT(2FI0.3)

$\mathrm{KI}=\mathrm{N} / 2$

$\mathrm{KIR}=0$

$\mathrm{KIS}=0$

30 DO $40 \mathrm{I}=\mathrm{I}, \mathrm{KI}-\mathrm{I}$ $\mathrm{W}(\mathrm{I})=\mathrm{X} 2(\mathrm{I})-\mathrm{X} 2(\mathrm{KI})$

$40 \quad \mathrm{Z}(\mathrm{I})=(\mathrm{Y}(\mathrm{I})-\mathrm{Y}(\mathrm{KI})) / \mathrm{W}(\mathrm{I})$ $\mathrm{W}(\mathrm{KI})=0$. 


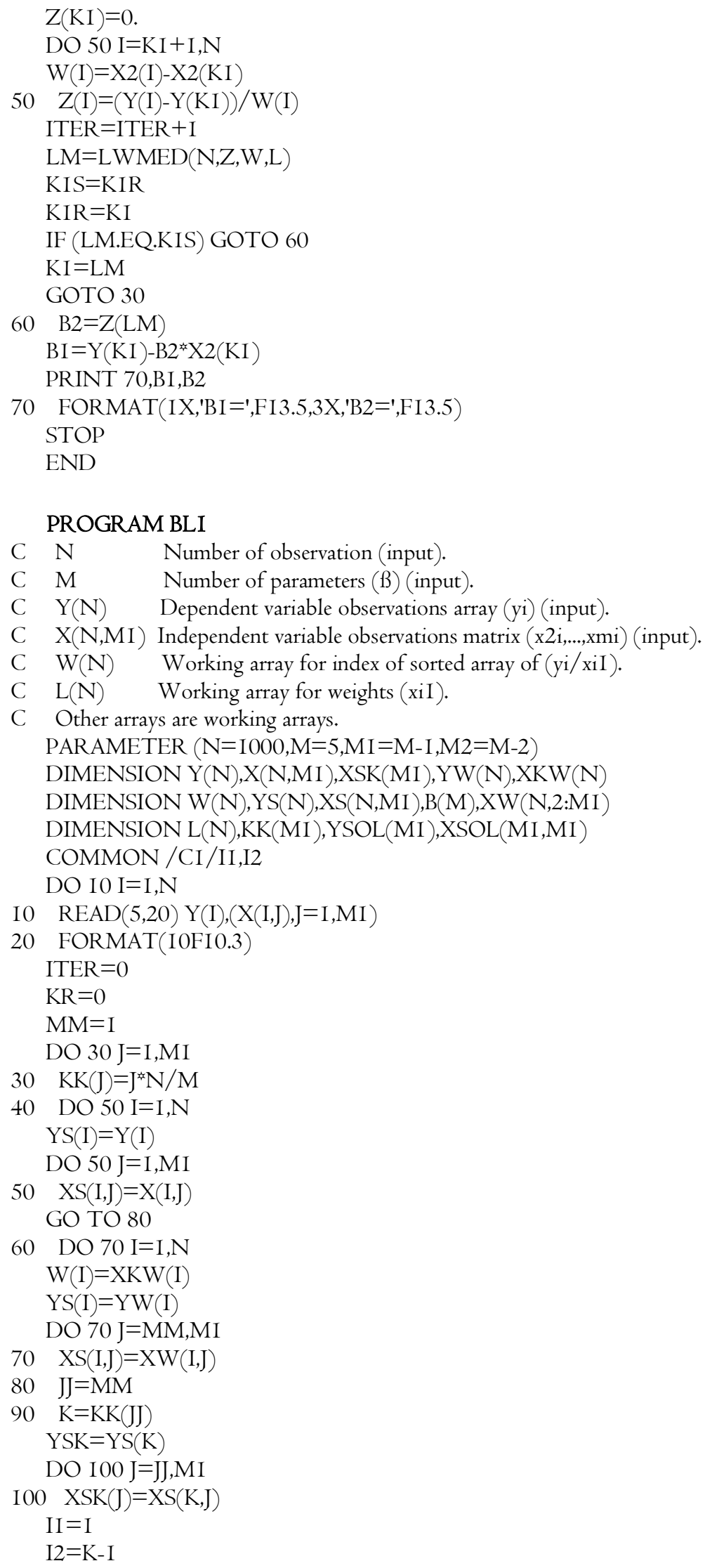


IIO DO I20 J=JJ,MI

I20 CALL COLI $(\mathrm{XSK}(\mathrm{J}), \mathrm{XS}(\mathrm{I}, \mathrm{J}))$

CALL COL2(YSK,JJ,W,YS,XS(I,JJ))

IF(I2.EQ.N) GO TO I30

$\mathrm{II}=\mathrm{K}+\mathrm{I}$

$\mathrm{I} 2=\mathrm{N}$

GO TO IIO

$\mathrm{I} 30 \mathrm{~W}(\mathrm{~K})=0$.

IF (JJ.EQ.MI) GO TO 190

$\mathrm{II}=\mathrm{I}$

$\mathrm{I} 2=\mathrm{K}-\mathrm{I}$

I40 DO I50 J=JJ+I,MI

I50 CALL COL3(XS(I,J),XS(I,JJ))

IF(I2.EQ.N) GO TO I60

$\mathrm{II}=\mathrm{K}+\mathrm{I}$

$\mathrm{I} 2=\mathrm{N}$

GO TO I40

I60 IF(JJ.NE.MM) GO TO I80

DO I70 I=I,N

$\mathrm{XKW}(\mathrm{I})=\mathrm{W}(\mathrm{I})$

$\mathrm{YW}(\mathrm{I})=\mathrm{YS}(\mathrm{I})$

DO I70 J=JJ+I,MI

$\mathrm{I} 70 \mathrm{XW}(\mathrm{I}, \mathrm{J})=\mathrm{XS}(\mathrm{I}, \mathrm{J})$

$180 \mathrm{JJ}=\mathrm{JJ}+\mathrm{I}$

GO TO 90

$190 \quad \mathrm{YS}(\mathrm{K})=0$.

ITER $=$ ITER $+\mathrm{I}$

$\mathrm{LM}=\mathrm{LWMED}(\mathrm{N}, \mathrm{YS}, \mathrm{W}, \mathrm{L})$

IF(LM.EQ.KR) GO TO 220

IOPT $=0$

200 IF(MM.EQ.MI) GO TO 210

$\mathrm{MM}=\mathrm{MM}+\mathrm{I}$

$\mathrm{KR}=\mathrm{KK}(\mathrm{MM})$

$\mathrm{KK}(\mathrm{MM})=\mathrm{LM}$

GO TO 60

2I0 $\mathrm{MM}=\mathrm{I}$

$\mathrm{KR}=\mathrm{KK}(\mathrm{MM})$

$\mathrm{KK}(\mathrm{MM})=\mathrm{LM}$

GO TO 40

220 IOPT $=$ IOPT $+\mathrm{I}$

IF (IOPT.NE.MI) GO TO 200

$\mathrm{B}(\mathrm{M})=\mathrm{YS}(\mathrm{LM})$

DO $230 \mathrm{I}=\mathrm{I}, \mathrm{MI}$

$\mathrm{YSOL}(\mathrm{I})=\mathrm{Y}(\mathrm{KK}(\mathrm{I}))$

DO $230 \mathrm{~J}=\mathrm{I}, \mathrm{MI}$

$230 \mathrm{XSOL}(\mathrm{I}, \mathrm{J})=\mathrm{X}(\mathrm{KK}(\mathrm{I}), \mathrm{J})$

$\mathrm{JJ}=\mathrm{I}$

$240 \quad \mathrm{YSK}=\mathrm{YSOL}(\mathrm{JJ})$

DO $250 \mathrm{~J}=\mathrm{JJ}, \mathrm{MI}$

$250 \mathrm{XSK}(\mathrm{J})=\mathrm{XSOL}(\mathrm{JJ}, \mathrm{J})$

DO $270 \mathrm{I}=\mathrm{JJ}, \mathrm{MI}$

IF(I.EQ.JJ) GO TO 270

$\mathrm{YSOL}(\mathrm{I})=\mathrm{YSOL}(\mathrm{I})-\mathrm{YSK}$

DO $260 \mathrm{~J}=\mathrm{JJ}, \mathrm{MI}$

$260 \mathrm{XSOL}(\mathrm{I}, \mathrm{J})=\mathrm{XSOL}(\mathrm{I}, \mathrm{J})-\mathrm{XSK}(\mathrm{J})$

YSOL(I) $=$ YSOL $(\mathrm{I}) / \mathrm{XSOL}(\mathrm{I}, \mathrm{JJ})$ 
270 CONTINUE

DO $290 \mathrm{I}=\mathrm{JJ}, \mathrm{MI}$

IF(I.EQ.JJ) GO TO 290

DO $280 \mathrm{~J}=\mathrm{JJ}+\mathrm{I}, \mathrm{MI}$

$280 \mathrm{XSOL}(\mathrm{I}, \mathrm{J})=\mathrm{XSOL}(\mathrm{I}, \mathrm{J}) / \mathrm{XSOL}(\mathrm{I}, \mathrm{JJ})$

290 CONTINUE

IF (JJ.EQ.M2) GO TO 300

$\mathrm{JJ}=\mathrm{JJ}+\mathrm{I}$

GO TO 240

300 DO $320 \mathrm{I}=\mathrm{I}, \mathrm{M} 2$

$\mathrm{K}=\mathrm{M}-\mathrm{I}$

$\mathrm{S}=\mathrm{YSOL}(\mathrm{K})$

DO 3 IO J=K,MI

$310 \mathrm{~S}=\mathrm{S}-\mathrm{B}(\mathrm{J}+\mathrm{I})^{*} \mathrm{XSOL}(\mathrm{K}, \mathrm{J})$

$320 \mathrm{~B}(\mathrm{~K})=\mathrm{S}$

$\mathrm{S}=\mathrm{Y}(\mathrm{KK}(\mathrm{I}))$

DO $330 \mathrm{~J}=\mathrm{I}, \mathrm{MI}$

$330 \mathrm{~S}=\mathrm{S}-\mathrm{B}(\mathrm{J}+\mathrm{I})^{*} \mathrm{X}(\mathrm{KK}(\mathrm{I}), \mathrm{J})$

$\mathrm{B}(\mathrm{I})=\mathrm{S}$

PRINT 340,(B(J),J=I,M)

340 FORMAT(IX,FI3.5)

PRINT 350,(KK(J),J=I,MI),LM,ITER

350 FORMAT(IX,II3)

STOP

END

SUBROUTINE COLI(VI,V2)

DIMENSION V2(I)

COMMON /CI/II,I2

DO I I $=\mathrm{II}, \mathrm{I} 2$

I V2(I) $=\mathrm{V} 2(\mathrm{I})-\mathrm{VI}$

RETURN

END

SUBROUTINE COL2(YSK,JJ,VI,YS,V2)

DIMENSION VI(I),V2(I),YS(I)

COMMON /CI/II,I2

IF (JJ.NE.I) GO TO 2

DO I I=II,I2

$\mathrm{VI}(\mathrm{I})=\mathrm{V} 2(\mathrm{I})$

I $\quad \mathrm{YS}(\mathrm{I})=(\mathrm{YS}(\mathrm{I})-\mathrm{YSK}) / \mathrm{V} 2(\mathrm{I})$

RETURN

2 DO $3 \mathrm{I}=\mathrm{II}, \mathrm{I} 2$

$\mathrm{VI}(\mathrm{I})=\mathrm{VI}(\mathrm{I})^{*} \mathrm{~V} 2(\mathrm{I})$

$3 \quad \mathrm{YS}(\mathrm{I})=(\mathrm{YS}(\mathrm{I})-\mathrm{YSK}) / \mathrm{V} 2(\mathrm{I})$

RETURN

END

SUBROUTINE COL3(VI,V2)

DIMENSION VI(I),V2(I)

COMMON /CI/II,I2

DO I I=II,I2

I $\quad \mathrm{VI}(\mathrm{I})=\mathrm{VI}(\mathrm{I}) / \mathrm{V} 2(\mathrm{I})$

RETURN

END 


\section{References}

Barrodale, F.D.K. Roberts (1974) Algorithm 478: Solution of an overdetermined system of equations in the $L_{1}$ norm. Commun. ACM, I7, 319- 320.

Bijan Bidabad (1987a) Least absolute error estimation. The First International Conference on Statistical Data Analysis Based on the Li norm and Related Methods, Neuchatel, Switzerland. http://www.bidabad.com/doc/lae-I.pdf

Bijan Bidabad (1987b) Least absolute error estimation, part II. Submitted to the First International Conference on Statistical Data Analysis Based on the $\mathrm{L}_{1}$ norm and Related Methods, Neuchatel, Switzerland. http://www.bidabad.com/doc/lae-II.pdf

Bijan Bidabad (I988a) A proposed algorithm for least absolute error estimation. Proc. of the Third Seminar of Mathematical Analysis. Shiraz Univ., 24-34, Shiraz, Iran.

Bijan Bidabad (I988b) A proposed algorithm for least absolute error estimation, part II. Proc. of the Third Seminar of Mathematical Analysis, Shiraz Univ., 35-50, Shiraz, Iran.

Bijan Bidabad (1989a) Discrete and continuous $\mathrm{L}_{1}$ norm regressions, proposition of discrete approximation algorithms and continuous smoothing of concentration surface, Ph.D. thesis, Islamic Azad Univ., Tehran, Iran. http://www.bidabad.com/doc/LI-norm-thesis-en.pdf

Bijan Bidabad (1989b) Discrete and continuous Li norm regressions, proposition of discrete approximation algorithms and continuous smoothing of concentration surface, Ph.D. thesis, Islamic Azad Univ., Tehran, Iran. Farsi translation. http://www.bidabad.com/doc/LI-norm-thesis-fa.pdf

Bijan Bidabad (2005). Li norm based computational algorithms. http://www.bidabad.com/doc/1I-article6.pdf

Bijan Bidabad (2005). Li norm solution of overdetermined system of linear equations. http://www.bidabad.com/doc/lIarticle5.pdf

Bijan Bidabad (2005). Li norm based data analysis and related methods. http://www.bidabad.com/doc/1I-articlI.pdf

Bijan Bidabad (2005). New algorithms for the Li norm regression. http://www.bidabad.com/doc/1I-article2.pdf

Bijan Bidabad (2005). Comparative study of the Li norm regression algorithms. http://www.bidabad.com/doc/1I-articl3.pdf

Bijan Bidabad (2005). Continuous Li norm estimation of Lorenz curve. http://www.bidabad.com/doc/1I-articl4.pdf

Bijan Bidabad (1993). Estimating Lorenz curve for Iran by using continuous $\mathrm{L}_{\mathrm{I}}$ norm estimation, Economics and Management Journal, Islamic Azad University, No. 19, winter I993, pp. 83-IOI. http://www.bidabad.com/doc/iraninc-lI.pdf

Bijan Bidabad (2005). Continuous Lı norm estimation of Lorenz curve when probability density function is known.

Bijan Bidabad (2005). USA Income distribution counter-business-cyclical trend (Estimating Lorenz curve using continuous $\mathrm{L}_{\mathrm{I}}$ norm estimation). First meeting of the Society for the Study of Economic Inequality (ECINEQ), Palma de Mallorca, Spain, July 20-22, 2005.

http://www.uib.es/congres/ecopub/ecineq/general.html

http://www.uib.es/congres/ecopub/ecineq/papers/039Bidabab.pdf

http://www.bidabad.com/doc/estimating-lorenz-us.pdf

Bijan Bidabad, Hamid Shahrestani. (2008) An implied inequality index using Li norm estimation of Lorenz curve. Global Conference on Business and Finance Proceedings. Mercedes Jalbert, managing editor, ISSN I93 I-0285 CD, ISSN I94I-9589 Online, Volume 3, Number 2, 2008, The Institute for Business and Finance Research, Ramada Plaza Herradura, San Jose, Costa Rica, May 28-3I, 2008, pp. I48-I63. Global Journal of Business Research, Vol. 4, No. I, 2010, pp.29-45.

http://www.bidabad.com/doc/LI-Implied-inequality-index-4.pdf

http://www.theibfr.com/archive/ISSN-I94I-9589-V3-N2-2008.pdf

http://www.bidabad.com/doc/SSRN-idI63186I.pdf

P. Bloomfield, W. Steiger (1980) Least absolute deviations curve fitting. SIAM J. Sci. Stat. Com.I,290-30I.

J. Chambers (I97I) Algorithm 4I0: partial sorting. Commun. ACM, I4, 357- 358.

C.A.R. Hoare (I96I) Algorithm 63 partition; 64, quicksort; and 65, find., Comm. ACM, 4, July, 32I -322.

C.A.R. Hoare (I962) Quicksort. Comput. J., 5, I0-I5. 
S. Scowen (I965) Algorithm 27I Quickersort. Commun. ACM, 8, 669-670.

R.S. Singleton (1969) Algorithm 347 Sort. Comm. ACM, I2, I85-I86.

\section{Copyrights}

Copyright for this article is retained by the author(s), with first publication rights granted to the journal. This is an open-access article distributed under the terms and conditions of the Creative Commons Attribution license (http://creativecommons.org/licenses/by/4.0/). 